\title{
Some expansions for a class of generalized Humbert matrix polynomials
}

\author{
H. M. Srivastava ${ }^{1,2} \cdot$ Waseem A. Khan ${ }^{3} \cdot$ Hiba Haroon ${ }^{3}$ \\ Received: 7 April 2019 / Accepted: 12 July 2019 / Published online: 24 July 2019 \\ (c) The Author(s) 2019
}

\begin{abstract}
The paper is an accomplishment of a new 3-variable 4-parameter generating function for Humbert matrix polynomials with an approach of unifying several classes of matrix valued polynomials using standard techniques of series manipulation. The results are contained in the form of explicit expression, hypergeometric matrix representation, generating functions and three additional expansions in nexus with Legendre, Hermite and Gegenbauer polynomials within discrete sections. A range of special cases is evenly traced that accounts due to the genuine wholesome generalization of such matrix polynomials.
\end{abstract}

Keywords Humbert matrix polynomials · Generalized hypergeometric series · Generating matrix functions $\cdot$ Generating relations

Mathematics Subject Classification $33 \mathrm{C} 25 \cdot 15 \mathrm{~A} 60 \cdot 33 \mathrm{C} 45 \cdot 33 \mathrm{E} 20$

\section{Introduction}

Special functions of matrices is a prominent topic in the literature of matrix analysis. A large piece of mathematics and its applications (both theoretical and practical) has been cut across the subject of orthogonal polynomials. The property of orthogonality, Rodrigues formula, a relation between different orthogonal matrix polynomials, matrix differential equation, a three-term matrix recurrence relation (see [5,9,10,29]) holds the theoretical examples, while, statistics, group representation theory, scattering theory, differential equations, Fourier series

$凶$ Hiba Haroon

hibaharoon786@gmail.com

H. M. Srivastava

harimsri@math.uvic.ca

Waseem A. Khan

waseem08_khan@rediffmail.com

1 Department of Mathematics and Statistics, University of Victoria, Victoria, BC V8W 3R4, Canada

2 Department of Medical Research, China Medical University Hospital, China Medical University, Taichung 40402, Taiwan, Republic of China

3 Department of Mathematics, Faculty of Science, Integral University, Lucknow 226026, India 
expansions, interpolation and quadrature and splines, (refer to $[4,7,11,17,18,21]$ ) embrace the practical ones. With passing years, this study has taken more systematic configuration due to the fact that many basic results of scalar orthogonality have been extended to the matrix frame. In [33], Srivastava and Brenner gave bounds for Jacobi and related polynomials derived by matrix methods. Latest innovations in matrix versions for the classical families of orthogonal polynomials such as Jacobi, extended Jacobi, Bessel, Hermite, Laguerre, Gegenbauer, Chebyshev polynomials and some other special functions are introduced by many authors for matrices in $C^{\mathbb{N} \times \mathbb{N}}$, (see for example [2,3,6-8,11-16,20,27-31]).

An organized study for the generalization of Humbert, Gegenbauer and several other polynomial systems is casted by Gould [12] using the generating function

$$
\left(c-m x t+y t^{m}\right)^{-p}=\sum_{n=0}^{\infty} P_{n}(m, x, y, p, c) t^{n},
$$

where $m$ is a positive integer, $|t|<1$ and rest parameters being generally unrestricted. The exact special cases of (1), including Gegenbauer, Legendre, Tchebycheff, Pincherle, Kinney and Humbert polynomials, are tabulated by Gould in [12]. $P_{n}(m, x, y, p, c)$ is defined explicitly by

$$
P_{n}(m, x, y, p, c)=\sum_{k=0}^{\left[\frac{n}{m}\right]}\left(\begin{array}{l}
p \\
k
\end{array}\right)\left(\begin{array}{l}
p-k \\
n-m k
\end{array}\right) c^{p-n+(m-1) k} y^{k}(-m x)^{n-m k} .
$$

In [28], the authors gave the matrix version for Gegenbauer polynomials through the generating function

$$
\left(1-2 x t+t^{2}\right)^{-A}=\sum_{n=0}^{\infty} C_{n}^{A}(x) t^{n} .
$$

The explicit formula being

$$
C_{n}^{A}(x)=\sum_{k=0}^{\frac{n}{2}} \frac{(-1)^{k}(A)_{n-k}(2 x)^{n-2 k}}{k !(n-2 k) !},
$$

where $A$ is positive stable matrix in the complex plane $C^{\mathbb{N} \times \mathbb{N}}$. In 1989, Sinha [32] gave the following generating relation

$$
\left[1-2 x t+t^{2}(2 x-1)\right]^{-v}=\sum_{n=0}^{\infty} S_{n}^{v}(x) t^{n},
$$

where

$$
S_{n}^{v}(x)=\sum_{k=0}^{\frac{n}{2}} \frac{(-1)^{k}(v)_{n-k}(2 x)^{n-2 k}(2 x-1)^{k}}{k !(n-2 k) !} .
$$

$S_{n}^{\nu}(x)$ is an interesting generalization of Shrestha polynomial $S_{n}(x)$ (see [31]).

Recently, Pathan et al. [23] studied a class of matrix polynomials associated with Humbert polynomials as an extension to the matrix framework of the classical families for the above mentioned polynomials by the relation

$$
\left(c-a x t+b t^{m}(2 y-1)\right)^{-A}=\sum_{n=0}^{\infty} P_{n, m}^{A}(x, y, a, b, c) t^{n} .
$$


He obtained the explicit representation for the polynomials as

$$
P_{n, m}^{A}(x, y, a, b, c)=\sum_{k=0}^{\frac{n}{m}} \frac{(-1)^{k} c^{-A-(n-(m-1) k) I}}{k !(n-m k) !}(a x)^{n-m k}[b(2 y-1)]^{k} .
$$

In the very next year, Pathan and Khan [24] generalized Sinha's generating relation (cf. 5)) to unify the generalized Humbert polynomials in two variables as

$$
\left[a-(b x+c y) t+d t^{m}(e x y-1)^{g}\right]^{-h}=\sum_{n=0}^{\infty} Q_{n, m, g, h}^{a, b, c, d, e}(x, y) t^{n},
$$

where $m \in \mathbb{N}, h>0$ and other parameters are unrestricted in general. Suitable selection of the parameters in the above expression generalize a number of polynomials studied by Agarwal and Parihar [1], Pathan and Khan [25], Gould [12], Milovanovic-Djordjevic [22] and Sinha [32] (also check [24]).

Motivated by the above literature, in the present article we give a 3-variable 4parameter matrix generalization for Humbert matrix polynomials $P_{n, m}^{A}(x, y, z ; a, b, c, d)$ [3V4PgHMaP] which unifies a number of matrix polynomials in the complex plane $C^{\mathbb{N} \times \mathbb{N}}$. The paper is organized as follows. In Sect. 2, some basel properties of the matrix functional calculus are given that will serve us throughout the presentation. In Sect. 3, we obtain two explicit formulae for the generalized Humbert matrix polynomials. Some special cases of proved fame are given in Sect. 4 and a generalized hypergeometric matrix series for $3 \mathrm{~V} 4 \mathrm{PgHMaP}$ is established in Sect. 5. In last two sections, the 3-variable matrix polynomial $P_{n, m}^{A}(x, y, z ; a, b, c, d)$ is exploited for some more generating functions and additional expansions.

\section{Preliminaries}

Throughout this paper, $D_{0}$ denotes the complex plane cut along the negative real axis and $\sigma(A)$ ( particularly known as spectrum of $A$ ), denotes the set of all eigenvalues of $A$. Here, $A$ is a positive stable matrix in the complex plane $C^{\mathbb{N} \times \mathbb{N}}$ if $\Re(\lambda)>0$ for all $\lambda \in \sigma(A)$.

Its two norm denoted by $\|A\|_{2}$ is defined by

$$
\|A\|_{2}=\sup _{x \neq 0} \frac{\|A x\|_{2}}{\|x\|_{2}}
$$

where for a vector $y$ in $\mathbb{C}^{N},\|y\|_{2}=\left(y^{T} y\right)^{1 / 2}$ is the Euclidean norm of $y$.

If $A$ is a matrix in $C^{\mathbb{N} \times \mathbb{N}}$ with $\sigma(A) \subset \Omega$, then from [8, p. 558], it follows that

$$
f(A) g(A)=g(A) f(A),
$$

where $f(z)$ and $g(z)$ are holomorphic functions of the complex variable $z$, which are defined in an open set $\Omega$ of the complex plane.

If

$$
A+n I \text { is invertible for every integer; } n \geq 0
$$

where $\mathrm{I}$ is the identity matrix in $\mathbb{C}^{N \times N}$, then it follows that

$$
(A)_{n}=\left\{\begin{array}{ll}
I, & n=0 \\
A(A+I) \ldots(A+(n-1) I), & n>0
\end{array}(\text { see }[13, \mathrm{p} .253])\right.
$$


and $(A)_{n}=\Gamma(A+n I) \Gamma^{-1}(A)$ due to $(12)$.

Thus from (13), one can desirable get (see [30, p. 30 (1.4) and p. 36 (4.2)]):

$$
\frac{(-1)^{k}}{(n-k) !} I=\frac{(-n)_{k}}{n !} I=\frac{(-n I)_{k}}{n !} ; \quad 0 \leq k \leq n
$$

and

$$
(-n I)_{2 k}=2^{2 k}\left(-\frac{1}{2} n I\right)_{k}\left(-\frac{1}{2}(n-1) I\right)_{k} .
$$

Definition 1 (Batahan [3]) For non-negative integers $p$ and $q$, the generalized hypergeometric matrix function is defined as:

$$
{ }_{r} F_{S}\left(A_{1}, \ldots, A_{r} ; B_{1}, \ldots, B_{s} ; z\right)=\sum_{n=0}^{\infty}\left(A_{1}\right)_{n} \ldots,\left(A_{r}\right)_{n}\left[\left(B_{1}\right)_{n}\right]^{-1} \ldots,\left[\left(B_{s}\right)_{n}\right]^{-1} \frac{z^{n}}{n !},
$$

where $A_{i}$ and $B_{j}$ are matrices in $C^{\mathbb{N} \times \mathbb{N}}$ such that the matrices $B_{j}(1 \leq j \leq s)$ satisfy the generic condition (12).

With $r=2$ and $s=1$ in (16), we get the hypergeometric matrix function ${ }_{2} F_{1}(A, B ; C ; z)$ (see [27]) of the type:

$$
{ }_{2} F_{1}(A, B ; C ; z)=\sum_{n=0}^{\infty} \frac{(A)_{n}(B)_{n}}{n !}\left[(C)_{n}\right]^{-1} z^{n} .
$$

Further with $r=1$ and $s=0$ in (16), one obtains the relation due to [19, p. 213]:

$$
(1-z)^{-A}=\sum_{n=0}^{\infty}(A)_{n} \frac{z^{n}}{n !}, \quad|z|<1 .
$$

The matrix power series in (17) is verified to be convergent by Ratio test for all complex number $z$.

Lemma $1[3,26,34]$ If $A(k ; n)$ and $B(k ; n)$ are matrices in $C^{N \times N}$ for $n \geq 0$ and $k \geq 0$, then the following relations are satisfied:

$$
\begin{aligned}
& \sum_{n=0}^{\infty} \sum_{k=0}^{n} A(k, n)=\sum_{n=0}^{\infty} \sum_{k=0}^{\left[\frac{n}{m}\right]} A(k, n-(m-1) k), \\
& \sum_{n=0}^{\infty} \sum_{k=0}^{\left[\frac{n}{m}\right]} A(k, n)=\sum_{n=0}^{\infty} \sum_{k=0}^{\infty} A(k, n+m k),
\end{aligned}
$$

and for $m=2$, we write

$$
\begin{aligned}
& \sum_{n=0}^{\infty} \sum_{k=0}^{\infty} B(k, n)=\sum_{n=0}^{\infty} \sum_{k=0}^{n} B(k, n-k), \\
& \sum_{n=0}^{\infty} \sum_{k=0}^{\left[\frac{n}{2}\right]} B(k, n)=\sum_{n=0}^{\infty} \sum_{k=0}^{\infty} B(k, n+2 k) .
\end{aligned}
$$

The above lemma provides results about double matrix series. The proof are analogous to that of the scalar case discussed in [26, p. 56] and [34, p. 101]. 
To meet the results, we will also use the following Binomial relation (see [26]).

$$
(\mu-v)^{n}=\sum_{n=0}^{\infty} \frac{n !(-1)^{k} \mu^{n-k} v^{k}}{k !(n-k) !} .
$$

\section{Some expansions for a class of generalized Humbert matrix polynomials}

Let A be a positive stable matrix in the complex plain $C^{N \times N}$, where, $C$ is the set of all complex numbers, for every Nth order square matrices. In view of (7) and (9), we define 3variable 4-parameter generalized Humbert matrix polynomials (3V4PgHMaP) with matrix generating relation:

$$
\left[c-(a x+b z) t+d t^{m}(2 z y-1)\right]^{-A}=\sum_{n=0}^{\infty} P_{n, m}^{A}(x, y, z ; a, b, c, d) t^{n},
$$

where, $m \in \mathbb{N}$ (set of natural numbers) and other parameters being unrestricted in general.

We shall regard the complicated notation $P_{n, m}^{A}(x, y, z ; a, b, c, d)$ by $\mathbb{P}(x, y, z)$ from now onwards in the paper.

In this section, we obtain two very interesting explicit formulae (power series) for 3V4PgHMaP $[\mathbb{P}(x, y, z)]$.

Theorem 1 For all $m \geq 2, \mathbb{P}(x, y, z)$ takes the following explicit forms:

$$
\begin{aligned}
\mathbb{P}(x, y, z)= & \sum_{k=0}^{[n / m]} \frac{(-1)^{k} c^{-A-(n-(m-1) k) I}(A)_{n-(m-1) k}}{k !(n-m k) !} \\
& \times(a x+b z)^{n-m k}[d(2 z y-1)]^{k},
\end{aligned}
$$

where $(A)_{n}$ is the general Pochhammer function in the matrix functional calculus $(c f .(13))$ for complex square matrix A.

And

$$
\begin{aligned}
\mathbb{P}(x, y, z)= & \sum_{s=0}^{\left[\frac{n}{m-1}\right]} \sum_{k=\left[\frac{\vartheta}{2}\right]}^{\infty} \frac{c^{-A-\vartheta I}(-1)^{n}(-(n-k))_{(m-1) s}(A)_{\vartheta-k}}{(n-k) ! s !(2 k-\vartheta) !} \\
& \times(2 A+2(\vartheta-k) I)_{2 k-\vartheta}\left\{-\frac{(a x+b z)}{2}\right\}^{\vartheta}\left\{\frac{4 d c(2 z y-1)}{(a x+b z)^{2}}\right\}^{s}, \\
= & \sum_{s=0}^{\left[\frac{n}{m-1}\right]} \sum_{k=\left[\frac{\vartheta}{2}\right]}^{\infty} \frac{c^{-A-\vartheta I}(-1)^{n}(-(n-k))_{(m-1) s}}{(n-k) ! s !(2 k-\vartheta) !} \frac{(2 A)_{\vartheta}}{2^{2 k}} \\
& \times\left[\left(A+\frac{1}{2} I\right)_{\vartheta-k}\right]^{-1}\left\{-\frac{(a x+b z)}{2}\right\}^{\vartheta}\left\{\frac{4 d c(2 z y-1)}{(a x+b z)^{2}}\right\}^{s}
\end{aligned}
$$

where $\vartheta=n-(m-2) s$.

Proof By using the relations (18) and (23), (24) can be expressed as

$$
\sum_{n=0}^{\infty} \mathbb{P}(x, y, z) t^{n}=c^{-A} \sum_{n=0}^{\infty} \frac{(A)_{n}}{n !}\left[\frac{(a x+b z) t}{c}-\frac{d t^{m}(2 z y-1)}{c}\right]^{n}
$$


or

$$
\begin{aligned}
& =c^{-A_{1} F_{0}}\left[\begin{array}{c}
A ; \\
-;
\end{array} \frac{(a x+b z) t}{c}-\frac{d t^{m}(2 z y-1)}{c}\right](\text { cf. Definition 1) } \\
& =c^{-A} \sum_{n=0}^{\infty} \frac{(A)_{n}}{n !} \sum_{k=0}^{n} \frac{n !(-1)^{k}}{k !(n-k) !}\left[\frac{(a x+b z) t}{c}\right]^{n-k}\left[\frac{d t^{m}(2 z y-1)}{c}\right]^{k} \\
& \times \sum_{n=0}^{\infty} \mathbb{P}(x, y, z) t^{n}=c^{-A} \sum_{n=0}^{\infty} \sum_{k=0}^{n} \frac{(-1)^{k}(A)_{n}}{k !(n-k) ! c^{n}}(a x+b z)^{n-k} \\
& \times[d(2 z y-1)]^{k} t^{n-k+m k} \text {. }
\end{aligned}
$$

By Lemma 1 (cf. (19)),

$$
\sum_{n=0}^{\infty} \sum_{k=0}^{n} A(k, n)=\sum_{n=0}^{\infty} \sum_{k=0}^{[n / m]} A(k, n-(m-1) k)
$$

the expression in (30), transforms as

$$
\begin{aligned}
\sum_{n=0}^{\infty} \mathbb{P}(x, y, z) t^{n}= & \sum_{n=0}^{\infty} \sum_{k=0}^{[n / m]} \frac{(-1)^{k} c^{-A-(n-(m-1) k) I}(A)_{n-(m-1) k}}{k !(n-m k) !} \\
& \times(a x+b z)^{n-m k}[d(2 z y-1)]^{k} t^{n},
\end{aligned}
$$

which on equating the coefficients of $t^{n}$ yields the explicit series (25) for $\mathbb{P}(x, y, z)$.

One can desirable get from (25) that

$$
\begin{aligned}
& P_{0, m}^{A}(x, y, z ; a, b, c, d)=C^{-A}, \\
& P_{1, m}^{A}(x, y, z ; a, b, c, d)= \begin{cases}C^{-A+I} A(a x+b z) ; & m>1 \\
C^{-A+I} A[a x+b z-d(2 z y-1)] ; & m=1\end{cases}
\end{aligned}
$$

and

$$
P_{n, m}^{A}(0,0, z ; a, b, c, d)=\frac{C^{-A+I}}{n !}(A)_{n} d^{n}
$$

Now, using a different approach on the generating relation (24), we get,

$$
\sum_{n=0}^{\infty} \mathbb{P}(x, y, z) t^{n}=c^{-A}\left[1-\frac{(a x+b z) t}{2 c}\right]^{-2 A}\left[1-\frac{\frac{(a x+b z)^{2} t^{2}}{4 c^{2}}-\frac{d t^{m}(2 z y-1)}{c}}{\left(1-\frac{(a x+b z) t}{2 c}\right)^{2}}\right]^{-A}
$$


which on using (18) and (23) in rhs of the above equation, gives

$$
\begin{aligned}
\sum_{n=0}^{\infty} & \mathbb{P}(x, y, z) t^{n}=c^{-A} \sum_{k=0}^{\infty} \frac{(A)_{k}}{k !}\left\{\frac{(a x+b z)^{2} t^{2}}{4 c^{2}}-\frac{d t^{m}(2 z y-1)}{c}\right\}^{k} \\
& \times \sum_{n=0}^{\infty} \frac{(-2 A-2 k)_{n}}{n !}\left\{\frac{(a x+b z) t}{2 c}\right\}^{n}, \\
= & \sum_{n, k=0}^{\infty} \sum_{s=0}^{k} \frac{c^{-A-(n-2 k) I}(-1)^{n}}{n ! s !(k-s) !}(2 A+2 k)_{n}\left\{\frac{(a x+b z)}{2}\right\}^{n+2 k} \\
& \times\left\{-\frac{4 d c(2 z y-1)}{(a x+b z)^{2}}\right\}^{s} t^{n+2 k+(m-2) s} .
\end{aligned}
$$

Now as $k \rightarrow k+s$ and applying Srivastava-Manocha identity [35, p. 100 (2)], we find

$$
\begin{aligned}
& \sum_{n=0}^{\infty} \mathbb{P}(x, y, z) t^{n}=\sum_{n, k, s=0}^{\infty} \frac{c^{-A-(n-2 k-2 s) I}(-1)^{n}}{n ! s ! k !}(2 A+2 k)_{n} \\
& \quad \times\left\{\frac{(a x+b z)}{2}\right\}^{n+2 k+2 s}\left\{-\frac{4 d c(2 z y-1)}{(a x+b z)^{2}}\right\}^{s} t^{n+2 k+m s} .
\end{aligned}
$$

A simple replacement $n=n-2 k-m s$ and comparison of coefficients of $t^{n}$ gives another explicit function (26) for $\mathbb{P}(x, y, z)$ as

$$
\begin{aligned}
\mathbb{P}(x, y, z)= & \sum_{s=0}^{\left[\frac{n}{m-1}\right]} \sum_{k=\left[\frac{\vartheta}{2}\right]}^{\infty} \frac{c^{-A-(\vartheta) I}(-1)^{n}(-(n-k))_{(m-1) s}(A)_{\vartheta-k}}{(n-k) ! s !(2 k-\vartheta) !} \\
& \times(2 A+2(\vartheta-k) I)_{2 k-\vartheta}\left\{-\frac{(a x+b z)}{2}\right\}^{\vartheta}\left\{\frac{4 d c(2 z y-1)}{(a x+b z)^{2}}\right\}^{s},
\end{aligned}
$$

where, $\vartheta=n-(m-2) s$.

In light of the relation,

$$
\begin{aligned}
& (A)_{n-k-(m-2) s}(2 A+2(n-k-(m-2) s) I)_{2 k-n+(m-2) s}=\frac{(2 A)_{n-(m-2) s}}{2^{2 k}} \\
& \times\left[\left(A+\frac{1}{2} I\right)_{n-k-(m-2) s}\right]^{-1} .
\end{aligned}
$$

Equation (33) can be rewritten as

$$
\begin{aligned}
& P_{n, m}^{A}(x, y, z ; a, b, c, d) \\
& =\sum_{s=0}^{\left[\frac{n}{m-1}\right]} \sum_{k=\left[\frac{n-(m-2) s}{2}\right]}^{\infty} \frac{C^{-A-(n-(m-2) s) I}(-1)^{n}(-(n-k))_{(m-1) s}}{(n-k) ! s !(2 k-n+(m-2) s) !} \frac{(2 A)_{n-(m-2) s}}{2^{2 k}} \\
& \quad \times\left[\left(A+\frac{1}{2} I\right)_{n-k-(m-2) s}\right]^{-1}\left\{-\frac{(a x+b z)}{2}\right\}^{n-(m-2) s}\left\{\frac{4 d c(2 z y-1)}{(a x+b z)^{2}}\right\}^{s},
\end{aligned}
$$

which coincides with our assertion (27). 


\section{Special cases of 3V4PgHMaP}

Upon assigning particular values to the parameters and variables we interestingly get a range of special cases for the generating Eq. (24) and for the relations (25)-(27), as discussed below:

1. Setting $b=0, z=1$ and replacing $d$ by $b$ in (24), we get the result of Pathan et al. [23] generated by

$$
\left[c-a x t+b t^{m}(2 y-1)\right]^{-A}=\sum_{n=0}^{\infty} P_{n, m}^{A}(x, y ; a, b, c) t^{n},
$$

where $P_{n, m}^{A}(x, y ; a, b, c)$ refers to the matrix polynomials associated with Humbert polynomials for two variables.

Same changes $(b=0, z=1, d=b)$ in (25) and (26), gives the explicit functions

$$
\begin{aligned}
P_{n, m}^{A}(x, y ; a, b, c)= & \sum_{k=0}^{[n / m]} \frac{(-1)^{k} c^{-A-(n-(m-1) k) I}(A)_{n+(1-m) k}}{k !(n-m k) !} \\
& \times(a x)^{n-m k}[b(2 y-1)]^{k},
\end{aligned}
$$

and

$$
\begin{aligned}
& P_{n, m}^{A}(x, y ; a, b, c) \\
& =\sum_{s=0}^{\left[\frac{n}{m-1}\right]} \sum_{k=\left[\frac{n-(m-2) s}{2}\right]}^{\infty} \frac{c^{-A-(n-(m-2) s) I}(-1)^{n}(-(n-k))_{(m-1) s}(A)_{n-(m-2) s-k}}{(n-k) ! s !(2 k-n+(m-2) s) !} \\
& \quad \times(2 A+2(n-(m-2) s-k) I)_{2 k-n+(m-2) s}\left\{-\frac{(a x)}{2}\right\}^{n-(m-2) s}\left\{\frac{4 b c(2 y-1)}{a^{2} x^{2}}\right\}^{s} .
\end{aligned}
$$

For proofs of (37) and (38) (see [23] p. 210).

2. Keeping $b=0=y, a=m, c=1=-d$ in (24) and (25), we get

$$
\left[1-m x t+t^{m}\right]^{-A}=\sum_{n=0}^{\infty} h_{n, m}^{A}(x) t^{n},
$$

and

$$
h_{n, m}^{A}(x)=\sum_{k=0}^{[n / m]} \frac{(-1)^{k}(A)_{n+(1-m) k}}{k !(n-m k) !}(m x)^{n-m k},
$$

where $h_{n, m}^{A}(x)$ is the matrix version of Humbert polynomials $h_{n, m}^{v}(x)$ for one variable [25].

3 . Further $m=3$ in (39) and (40), gives us

$$
\left[1-3 x t+t^{3}\right]^{-A}=\sum_{n=0}^{\infty} h_{n}^{A}(x) t^{n},
$$


where $h_{n}^{A}(x)$ is the matrix generating relation of Pincherle polynomials $h_{n}(x)$ (see [22,25]), with finite series representation as

$$
h_{n}^{A}(x)=\sum_{k=0}^{[n / 3]} \frac{(-1)^{k}(A)_{n-2 k}(3 x)^{n-3 k}}{k !(n-3 k) !} .
$$

4. Since

$$
P_{n, 2}^{A}(x, 0, z ; 2,-1,1,-1)=C_{n}^{A}(x), \quad(\text { see }[29, \text { p. 109(40)]). }
$$

Therefore, the adjustment $m=a=2, y=0$, and $c=1=-d=-b$ in (24) and (27), gives us another known result [29]

$$
\begin{aligned}
& {\left[1-2 x t+t^{2}\right]^{-A}=\sum_{n=0}^{\infty} C_{n}^{A}(x) t^{n},} \\
& C_{n}^{A}(x)=(2 A)_{n} \sum_{k=0}^{[n / 2]} \frac{1}{2^{2 k} k !(n-2 k) !}\left[\left(A+\frac{1}{2} I\right)\right]^{-1}\left(x^{2}-1\right)_{k} x^{n-2 k}
\end{aligned}
$$

where $C_{n}^{A}(x)$ is the Gegenbauer matrix polynomial.

5. By setting $A=1, y=b=0, a=\sqrt{2 B}, c=1$ and $d=-1$ in (24), we get

$$
P_{n, 2}^{[1]_{1 \times 1}}(x, 0, z ; \sqrt{2 B}, 0,1,-1)=U_{n}(x, B),
$$

where, $U_{n}(x, B)$ is the Chebyshev matrix polynomials of second kind [2], defined by

$$
U_{n}(x, B)=\sum_{r=0}^{[n / 2]} \frac{(-1)^{r}(n-r) !(\sqrt{2 B} x)^{n-2 r}}{r !(n-2 r) !} .
$$

6. Further as $A \rightarrow \frac{1}{2}$ in (45), we get a class of Legendre matrix polynomials [27].

$$
P_{n, 2}^{\left[\frac{1}{2}\right]_{1 \times 1}}(x, 0, z ; \sqrt{2 B}, 0,1,-1)=P_{n}(x, B)
$$

where,

$$
P_{n}(x, B)=\sum_{r=0}^{[n / 2]} \frac{(-1)^{r}(2 n-2 r) !(\sqrt{2 B} x)^{n-2 r}}{2^{2 n-2 r} r !(n-r) !(2-2 r) !} .
$$

\section{Generalized hypergeometric matrix representation for $\mathbb{P}(x, y, z)$}

Here, we establish the matrix representation for 3V4PgHMaP in terms of generalized hypergeometric series.

Theorem 2 The following hypergeometric representation for $3 \operatorname{VP} \operatorname{PHMaP}, \mathbb{P}(x, y, z)$ holds well:

$$
\mathbb{P}(x, y, z)=\frac{(A)_{n} C^{-A-n I}}{n !}{ }_{m} F_{m-1}\left[\begin{array}{cl}
\Delta(m ;-n I) & ; \\
\Delta(m-1 ;-A-(n-1) I) & ;
\end{array}\right]
$$

where $W=\left(\frac{c}{m-1}\right)^{m-1}\left(\frac{m}{(a x+b z)}\right)^{m}[d(2 z y-1)]$, 
and $\Delta(n ; b I)$ refer to the array of $n$ parameters as $\frac{b I}{n}, \frac{(b+1) I}{n}, \ldots, \frac{(b+m-1) I}{n}$.

Proof For non-negative integers $n, m$, from (13) and (15) one can write

$$
(-n I)_{m k}=(-1)^{m k} \frac{n !}{(n-m k) !} I=m^{m k} \prod_{i=1}^{m}\left(\frac{-n+i-1}{m}\right)_{k}
$$

and

$$
(A)_{n-(m-1) k}=(-1)^{(m-1) k}(A)_{n}\left[(m-1)^{(m-1) k} \prod_{j=1}^{m-1}\left(\frac{(-A-n I)+j I}{m-1}\right)_{k}\right]^{-1} .
$$

We begin with the explicit expression (25) for $\mathbb{P}(x, y, z)$ :

$$
\begin{aligned}
\mathbb{P}(x, y, z)= & \sum_{k=0}^{[n / m]} \frac{(-1)^{k} C^{-A-(n-(m-1) k) I}(A)_{n-(m-1) k}}{k !(n-m k) !} \\
& \times(a x+b z)^{n-m k}[d(2 z y-1)]^{k} .
\end{aligned}
$$

Using (50) and (51) in (52), we get

$$
\begin{aligned}
\mathbb{P}(x, y, z)= & \sum_{k=0}^{[n / m]} \frac{(-1)^{m k} n !}{(n-m k) !} \frac{(A)_{n-(m-1) k}(-1)^{k}}{n !(-1)^{m k} k !} \frac{c^{-A-n I}(a x+b z)^{n}[d(2 z y-1)]^{k}}{c^{(1-m) k}(a x+b z)^{m k}}, \\
= & \sum_{k=0}^{\infty} m^{m k} \prod_{i=1}^{m}\left(\frac{-n+i-1}{m} I\right)_{k} \frac{(A)_{n}}{(m-1)^{(m-1) k} n ! k !}\left[\prod_{j=1}^{m-1}\left(\frac{-A-n I+j I}{m-1}\right)_{k}\right]^{-1} \\
& \times \frac{c^{-A-n I}}{c^{-(m-1) k}} \frac{(a x+b z)^{n}[d(2 z y-1)]^{k}}{(a x+b z)^{m k}}, \\
= & \frac{(A)_{n} C^{-A-n I}}{n !}(a x+b z)^{n} \sum_{k=0}^{\infty} \prod_{i=1}^{m}\left(\frac{-n+i-1}{m} I\right)_{k}\left[\prod_{j=1}^{m-1}\left(\frac{-A-n I+j I}{m-1}\right)_{k}\right]^{-1} \\
& \times \frac{c^{(m-1) k} m^{m k}}{k !(m-1)^{(m-1) k}} \frac{[d(2 z y-1)]^{k}}{(a x+b z)^{m k}} .
\end{aligned}
$$

Now, from (20), one easily gets the hypergeometric matrix expansion for generalized Humbert matrix polynomial $\mathbb{P}(x, y, z)$ precisely as

$$
\mathbb{P}(x, y, z)=\frac{(A)_{n} C^{-A-n I}}{n !}{ }_{m} F_{m-1}\left[\begin{array}{cl}
\Delta(m ;-n I) & ; \\
\Delta(m-1 ;-A-(n-1) I) & ;
\end{array}\right]
$$

where $W=\left(\frac{c}{m-1}\right)^{m-1}\left(\frac{m}{(a x+b z)}\right)^{m}[d(2 z y-1)]$,

and $\Delta(n ; b I)$ refer to the array of $n$ parameters as $\frac{b I}{n}, \frac{(b+1) I}{n}, \ldots, \frac{(b+m-1) I}{n}$.

We mark that, $A+n I$ and $\frac{-A-(n-m+1) I}{m-1}$ are invertible matrices. 
Remark 1 Substituting $z=1$ and $b=0$ in Theorem 2, we readily find a known result as

$$
\begin{aligned}
P_{n, m}^{A}(x, y ; a, c, d)= & \frac{(A)_{n} C^{-A-n I}}{n !}(a x)^{n}{ }_{m} F_{m-1}\left[\frac{-n I}{m}, \frac{(-n+1) I}{m}, \ldots, \frac{(-n+m-1) I}{m} ;\right. \\
& \left.\frac{-A-(n-1) I}{m-1}, \ldots, \frac{-A-(n-m+1) I}{m-1} ; \frac{c^{m-1} m^{m}[d(2 y-1)]}{(m-1)^{(m-1)}(a x)^{m}}\right] .
\end{aligned}
$$

The relationship (54) is already proved by Pathan et al. (see [23]).

Remark 2 Choosing the parameters $b=0=y, a=m$ and $c=-d=1$ in Theorem 2, we get the matrix representation (see [23]):

$$
\begin{aligned}
& h_{n, m}^{A}(x)=\frac{(A)_{n}(m x)^{n}}{n !}{ }_{m} F_{m-1}\left[\frac{-n I}{m}, \frac{(-n+1) I}{m}, \ldots, \frac{(-n+m-1) I}{m} ; \frac{-A-(n-1) I}{m-1},\right. \\
& \left.\ldots \frac{-A-(n-m+1) I}{m-1} ; \frac{1}{(m-1)^{(m-1)}(x)^{m}}\right],
\end{aligned}
$$

which reduces (49) in one variable. Further restricting $m=3$ in (55), we get $h_{n, m}^{A}(x)=$ $h_{n}^{A}(x)$ :

$$
h_{n}^{A}(x)=\frac{(A)_{n}(3 x)^{n}}{n !}{ }_{3} F_{2}\left[\frac{-n}{3} I, \frac{1-n}{3} I, \frac{2-n}{3} I ; \frac{1}{2}(I-A-n I), \frac{1}{2}(2 I-A-n I) ; \frac{1}{4 x^{3}}\right] \text {. }
$$

The relationship (56) is already proved by Khammasha and Shehata in [22].

Remark 3 The relationship $P_{n, 2}^{A}(x, 0, z ; 2,-1,1,-1)=C_{n}^{A}(x)$, descends (49) to the hypergeometric function ${ }_{2} F_{1}[-,-;-; x]$ of Sayyed et al. [29] for Gegenbaure matrix polynomials.

$$
C_{n}^{A}(x)=\frac{(A)_{n}(2 x)^{n}}{n !}{ }_{2} F_{1}\left[\frac{-n I}{m}, \frac{(1-n) I}{m} ; I-A-n I ; x^{-2}\right]
$$

\section{Some more generating functions}

Relying on a similar procedure as used in the previous section, we come across some more generating relations derived below. In the due course, we observe that these generating relations form the extended matrix versions for Sinha, Sheshtha, Pincherle, Kinney, Gegenbaure and Horadam-Pethe polynomials (see [15,16,22,27,32]).

Theorem 3 Let $A \in C^{\mathbb{N} \times \mathbb{N}}$ be a positive stable matrix, then for 3 V4PgHMaP the following generating relation holds true.

$$
\begin{aligned}
& \mathbb{P}(x, y, z)\left[(A)_{n}\right]^{-1}=\frac{c^{-A-n I}}{n !}[(a x+b z) t]^{n}{ }_{1} F_{m}\left[A+n I ; \frac{A+n I}{m}, \frac{A+(n-1) I}{m}, \ldots\right. \\
& \left.\frac{A+(n-m+1) I}{m} ; \frac{-d t^{m}(2 z y-1)}{c m^{m}}\right]
\end{aligned}
$$

where, $\frac{A+n I}{m}, \frac{A+(n-1) I}{m}, \ldots, \frac{A+(n-m+1) I}{m}$ are invertible matrices in $C^{\mathbb{N} \times \mathbb{N}}$. 
Proof We proceed by considering the explicit series (25)

$$
\begin{aligned}
& \sum_{n=0}^{\infty} \mathbb{P}(x, y, z)\left[(A)_{n}\right]^{-1} t^{n}=\sum_{n=0}^{\infty} \sum_{k=0}^{[n / m]} \frac{(-1)^{k}\left[(A)_{n}\right]^{-1} c^{-A-(n-(m-1) k) I}(A)_{n-(m-1) k}}{k !(n-m k) !} \\
& \quad \times[(a x+b z)]^{n-m k}[d(2 y z-1)]^{k} t^{n},
\end{aligned}
$$

which on using (50) and the relation $(A)_{n+k}=(A)_{n}(A+n I)_{k}$ gives

$$
\begin{aligned}
& \sum_{n=0}^{\infty} \mathbb{P}(x, y, z)\left[(A)_{n}\right]^{-1} t^{n}=\sum_{n=0}^{\infty} \sum_{k=0}^{[n / m]} \frac{(-1)^{k} c^{-A-(n-(m-1) k) I}}{k !(n-m k) !}(-1)^{m k}(A+(n-m k) I)_{k} \\
& \quad \times\left[m^{m k} \prod_{j=1}^{m}\left(\frac{(-A-n I)+j I}{m}\right)_{k}\right]^{-1}(a x+b z)^{n-m k}[d(2 z y-1)]^{k} t^{n} .
\end{aligned}
$$

Now, we apply (20) to get

$$
\begin{aligned}
& \sum_{n=0}^{\infty} \mathbb{P}(x, y, z)\left[(A)_{n}\right]^{-1} t^{n}=\sum_{n=0}^{\infty} \sum_{k=0}^{\infty} \frac{c^{-A-n I}}{k ! n ! c^{k} m^{m k}}\left[\prod_{j=0}^{m}\left(\frac{(A+n I)-j I}{m}\right)_{k}\right]^{-1} \\
& \quad \times(A+n I)_{k}(a x+b z)^{n}\left[d t^{m}(2 z y-1)\right]^{k} t^{n}
\end{aligned}
$$

or in view of (16), can be presented as

$$
\begin{aligned}
\sum_{n=0}^{\infty} \mathbb{P}(x, y, z)\left[(A)_{n}\right]^{-1} t^{n} \\
=\sum_{n=0}^{\infty} \frac{c^{-A-n I}}{n !}[(a x+b z) t]^{n} \\
\quad \times{ }_{1} F_{m}\left[A+n I ; \frac{A+(n-1) I}{m}, \frac{A+(n-2) I}{m}, \ldots, \frac{A+(n-m+1) I}{m} ; \frac{-d t^{m}(2 z y-1)}{c m^{m}}\right] .
\end{aligned}
$$

This meets our assertion (58).

Theorem 4 Let $A, B \in C^{\mathbb{N} \times \mathbb{N}}$ be positive stable matrix with $A B=B A$, then for $3 \mathrm{~V} 4 \mathrm{PgHMaP}$, the following generating relation holds true.

$$
\begin{aligned}
& \sum_{n=0}^{\infty}(B)_{n} P_{n, m}^{A}(x, y, z ; a, b, c, d)\left[(A)_{n}\right]^{-1} t^{n}=\sum_{n=0}^{\infty} \frac{c^{-A-n I}[(a x+b z) t]^{n}(B)_{n}}{n !} \\
& \quad \times_{m+1} F_{m}\left[A+n I ; \frac{B+n I}{m}, \frac{B+(n-1) I}{m}, \ldots \frac{B+(n+m-1) I}{m} ; \frac{A+n I}{m},\right. \\
& \left.\frac{A+(n-1) I}{m}, \ldots \frac{A+(n+m-1) I}{m} ; \frac{-d t^{m}(2 z y-1)}{c}\right] .
\end{aligned}
$$

Proof We begin with the explicit series

$$
\begin{aligned}
& \sum_{n=0}^{\infty}(B)_{n} \mathbb{P}(x, y, z)\left[(A)_{n}\right]^{-1} t^{n}=\sum_{n=0}^{\infty} \sum_{k=0}^{[n / m]} \frac{(-1)^{k}(B)_{n}\left[(A)_{n}\right]^{-1} c^{-A-(n-(m-1) k) I}}{k !(n-m k) !} \\
& \quad \times(A)_{n-(m-1) k}[(a x+b z)]^{n-m k}[d(2 y z-1)]^{k} t^{n},
\end{aligned}
$$


and redo the steps as in Theorem 3, to get generating function (61).

Working on same lines as in previous theorems, this time starting with (27) and using (20), we get two more relations. We state next two theorems without proof.

Theorem 5 Let $A \in C^{\mathbb{N} \times \mathbb{N}}$ be a positive stable matrix, then for 3 V4PgHMaP, the following generating relation holds true.

$$
\begin{aligned}
& \sum_{n=0}^{\infty} \mathbb{P}(x, y, z)\left[(2 A)_{n}\right]^{-1} t^{n}=\sum_{n=0}^{\infty} \sum_{s=0}^{\infty} \sum_{k=\left[\frac{n+s}{2}\right]}^{\infty} \frac{(-1)^{n+k} c^{-A}(-2 k)_{n+s}(-n)_{k}}{2^{2 k} n !(2 k) ! s !} \\
& \quad \times\left[\left(A+\frac{1}{2} I\right)_{n-k+s}(2 A+(n-s) I)_{m s}\right]^{-1}\left[\frac{(a x+b z) t}{2 C}\right]^{n}\left[\frac{2 d t^{m-1}(2 z y-1)}{a x+b z}\right]^{s} .
\end{aligned}
$$

Theorem 6 Let $A, B \in C^{\mathbb{N} \times \mathbb{N}}$ be a positive stable matrix, then for 3 V4PgHMaP, the following generating relation holds true.

$$
\begin{aligned}
\sum_{n=0}^{\infty} & (B)_{n} \mathbb{P}(x, y, z)\left[(2 A)_{n}\right]^{-1} t^{n}=\sum_{n=0}^{\infty} \sum_{s=0}^{\infty} \sum_{k=\left[\frac{n+s}{2}\right]}^{\infty} \frac{(-1)^{n+k} C^{-A}(-2 k)_{n+s}(-n)_{k}}{2^{2 k} n !(2 k) ! s !} \\
\times & {\left[\left(A+\frac{1}{2} I\right)_{n-k+s}(2 A+(n-s) I)_{m s}\right]^{-1}(B)_{n+(m-1) s}\left[\frac{(a x+b z) t}{2 C}\right]^{n} } \\
\times & {\left[\frac{2 d t^{m-1}(2 z y-1)}{a x+b z}\right]^{s} . }
\end{aligned}
$$

\section{Some additional expansions}

We recall the known algebraic polynomial expansions due to Ranville (see [26, p. 181 (4), p. 283 (4), p. 207 (2)]),

$$
\frac{(a x)^{n}}{n !}=\sum_{s=0}^{\left[\frac{n}{2}\right]} \frac{2 n-4 s+1}{s !\left(\frac{3}{2}\right)_{n-s}} P_{n-2 s}\left(\frac{a x}{2}\right),
$$

where $P_{n}(x)$ is Legendre polynomials.

$$
\frac{(2 x)^{n}}{n !}=\sum_{k=0}^{\left[\frac{n}{2}\right]} \frac{(v+n-2 k)}{k !(v)_{n+1-k}} C_{n-2 k}^{v}(x),
$$

where $C_{n}(x)$ is Gegenbaure polynomials.

and

$$
\frac{x^{n}}{n !}=\sum_{k=0}^{\left[\frac{n}{2}\right]} \frac{H_{n-2 k}(x)}{2^{n} k !(n-2 k) !},
$$

where $H_{n}(x)$ is Hermite polynomials.

In this section, we derive some additional expansions for the matrix polynomial $P_{n, m}^{A}(x, y, z ; a, b, c, d)$ using the explicit expression (27) in series of Legendre, Hermite and Gegenbaure polynomials. 


\section{Expansion 7.1.}

$$
\begin{aligned}
\mathbb{P}(x, y, z)= & \sum_{s=0}^{\infty} \sum_{k=\left[\frac{n+s}{2}\right]}^{\infty} \sum_{j=0}^{\left[\frac{n+s}{2}\right]} \frac{c^{-A-n I}(-1)^{n+k}(-n)_{k}(-2 k)^{n+s}(n+1)_{s}}{s ! j !(2 k) ! 2^{2 k}(v)_{n+s-j+1}} \\
& \times[2(n+s)-4 j+1](2 A)_{n+s}\left[\left(A+\frac{1}{2} I\right)_{n+s-k}\right]^{-1} \\
& \times\left(\frac{4 d c(2 z y-1)}{(a x+b z)^{2}}\right)^{s} P_{n+s-2 j}\left(\frac{a x+b z}{4}\right) .
\end{aligned}
$$

Expansion 7.2.

$$
\begin{aligned}
\mathbb{P}(x, y, z)= & \sum_{s=0}^{\infty} \sum_{k=\left[\frac{n+s}{2}\right]}^{\infty} \sum_{j=0}^{\left[\frac{n+s}{2}\right]} \frac{c^{-A-n I}(-1)^{n+k}(-n)_{k}(-2 k)^{n+s}(n+1)_{s}}{s ! j !(2 k) ! 2^{2 k}\left(\frac{3}{2}\right)_{n+s-j}} \\
& \times[v+n+s-2 j](2 A)_{n+s}\left[\left(A+\frac{1}{2} I\right)_{n+s-k}\right]^{-1} \\
& \times\left(\frac{4 d c(2 z y-1)}{(a x+b z)^{2}}\right)^{s} C_{n+s-2 j}^{v}\left(\frac{a x+b z}{4}\right) .
\end{aligned}
$$

\section{Expansion 7.3.}

$$
\begin{aligned}
\mathbb{P}(x, y, z)= & \sum_{s=0}^{\infty} \sum_{k=\left[\frac{n+s}{2}\right]}^{\infty} \sum_{j=0}^{\left[\frac{n+s}{2}\right]} \frac{c^{-A-n I}(-1)^{n+k+j}(-n)_{k}(-2 k)^{n+s}(n+1)_{s}}{s ! j !(2 k) ! 2^{n+2 k}(n+s-2 j) !} \\
& \times(2 A)_{n+s}\left[\left(A+\frac{1}{2} I\right)_{n+s-k}\right]^{-1}\left(\frac{4 d c(2 z y-1)}{(a x+b z)^{2}}\right)^{s} H_{n+s-2 j}\left(\frac{a x+b z}{2}\right) .
\end{aligned}
$$

Derivation of Expansions (7.1)-(7.3)

From (65), we can write

$$
\begin{aligned}
& \left(\frac{a x+b z}{2}\right)^{n-(m-2) s}=\sum_{j=0}^{\left[\frac{n-(m-2) s}{2}\right]} \frac{[2(n-(m-2) s)-4 j+1](n-(m-2) s) !}{j !\left(\frac{3}{2}\right)_{n-(m-2) s-j}} \\
& \times P_{n-(m-2) s-2 j}\left(\frac{a x+b z}{4}\right) .
\end{aligned}
$$

Now using (71) in (27), we get

$$
\begin{aligned}
\mathbb{P}(x, y, z)= & \sum_{s=0}^{\left[\frac{n}{m-1}\right]} \sum_{k=\left[\frac{n-(m-2) s}{2}\right]}^{\infty} \sum_{j=0}^{\left[\frac{n-(m-2) s}{2}\right]} \frac{c^{-A-(n-(m-2) s) I}(-(n-k))_{(m-1) s}}{(-1)^{m s}(2 k-n+(m-2) s) !} \\
& \times \frac{(2 A)_{n-(m-2) s}[2(n-(m-2) s)-4 j+1](n-(m-2) s) !}{s ! j !(n-k) ! 2^{2 k}\left(\frac{3}{2}\right)_{n-(m-2) s-j}} \\
& \times\left[\left(\left(A+\frac{1}{2}\right) I\right)_{n-k-(m-2) s}\right]^{-1}\left(\frac{4 d c(2 z y-1)}{(a x+b z)^{2}}\right)^{s} P_{n-(m-2) s-2 j}\left(\frac{a x+b z}{4}\right)
\end{aligned}
$$


which on applying (20), yields Expansion 7.1.

Likewise, modifying (66) and (67) for $x=a x+b z$ and then inserting in (27), we can easily get expansions 7.2 and 7.3.

\section{Compliance with ethical standards}

Conflict of interest The authors declare that they have no competing interests.

Open Access This article is distributed under the terms of the Creative Commons Attribution 4.0 International License (http://creativecommons.org/licenses/by/4.0/), which permits unrestricted use, distribution, and reproduction in any medium, provided you give appropriate credit to the original author(s) and the source, provide a link to the Creative Commons license, and indicate if changes were made.

\section{References}

1. Agarwal, R., Parihar, H.S.: On certain generalized polynomial system associated with Humbert polynomials. Sci. Ser. A Math. Sci. 23, 31-44 (2012)

2. Batahan, R.S.: A new extension of Hermite matrix polynomials and its applications. Linear Algebra Appl. 419(1), 82-92 (2006)

3. Batahan, R.S.: Generalized Gegenbauer matrix polynomials, series expansion and some properties. In: Ling, G.D. (ed.) Linear Algebra Research Advances, pp. 291-305. Nova Science Publishers, Hauppauge (2007)

4. Defez, E., Hervas, A., Law, A., Villanueva-Oller, J., Villanueva, R.J.: Progressive transmission of images: PC-based computations, using orthogonal matrix polynomials. Math. Comput. Model. 32(10), 1125-1140 (2000)

5. Defez, E., Jodar, L., Law, A., Ponsoda, E.: Three-term recurrences and matrix orthogonal polynomials. Util. Math. 57, 129-146 (2000)

6. Defez, E., Jodar, L., Law, A.: Jacobi matrix differential equation, polynomial solutions and their properties. Comput. Math. Appl. 48, 789-803 (2004)

7. Defez, E., Law, A., Villanueva-Oller, J., Villanueva, R.J.: Matrix cubic splines for progressive 3D imaging. J. Math. Imaging Vis. 17(1), 41-53 (2002)

8. Dunfold, N., Schwartz, J.: Linear Operators, Part I. Interscience, New York (1958)

9. Duran, A.J.: On orthogonal polynomials with respect to a positive definite matrix of measures. Can. J. Math. 47(1), 88-112 (1995)

10. Duran, A.J., Lopez-Rodriguez, P.: Orthogonal matrix polynomials: zeros and Blumenthal's theorem. J. Approx. Theory 84(1), 96-118 (1996)

11. Geronimo, J.S.: Scattering theory and matrix orthogonal polynomials on the real line. Circuits Syst. Signal Process. 1(3-4), 471-494 (1982)

12. Gould, H.W.: Inverse series relations and other expansions involving Humbert polynomials. Duke Math. J. 32(4), 697-711 (1965)

13. Hille, E.: Lectures on Ordinary Differential Equations. Addison-Wesley, New York (1968)

14. Horadan, A., Pethe, S.: Gegenbauer polynomials revisited. Fibonacci Q. 23, 295-299 (1985)

15. Horadan, A., Pethe, S.: Polynomials associated with Gegenbauer polynomials. Fibonacci Q. 19, 393-398 (1981)

16. Humbert, P.: Some extentions of Pincherle's polynomials. Proc. Edinb. Math. Soc. 39, 21-24 (1921)

17. James, A.T.: Special functions of matrix and single argument in statistics. In: Theory and Applications of Special Functions, pp. 497-520 (1975). https://doi.org/10.1016/B978-0-12-064850-4.50016-1

18. Jodar, L., Company, R., Ponsoda, E.: Orthogonal matrix polynomials and systems of second order differential equations. Differ. Equ. Dyn. Syst. 3, 269-288 (1996)

19. Jodar, L., Defez, E.: On Hermite matrix polynomials and Hermite matrix function. Approx. Theory Appl. 14(1), 36-48 (1998)

20. Jodar, L., Defez, E., Ponsoda, E.: Matrix quadrature and orthogonal matrix polynomials. Congr. Numer. 106, 141-153 (1995)

21. Milovanovic, G.V., Djordjevic, G.B.: On Some properties of Humbert's polynomials II. Facta Univ. Ser. Math. Inform. 6, 23-30 (1991)

22. Khammash, G.H., Shehata, A.: On Humbert matrix polynomials. Asian J. Curr. Eng. Math. 1(5), 232-240 (2012) 
23. Pathan, M.A., Bin-Saad, M.G., Al-Sarahi, F.: On matrix polynomials associated with Humbert polynomials. J. Korean Math. Soc. 21(3), 207-218 (2014)

24. Pathan, M.A., Khan, N.U.: A unified presentation of a class of generalized Humbert polynomials in two variables. ROMAI J. 11(2), 185-199 (2015)

25. Pathan, M.A., Khan, M.A.: On polynomials associated with Humbert's polynomials. Publ. Inst. Math. (Beograd) 62, 53-62 (1997)

26. Rainville, E.D.: Special Function. Publications de l'Intitut Mathematique. Macmillan Company, New York (1960)

27. Sastre, J., Defez, E., Jodar, L.: Laguerre matrix polynomials series expansion: theory and computer applications. Math. Comput. Model. 44, 1025-1043 (2006)

28. Sayyed, K.A., Metwally, M.S., Batahan, R.S.: Gegenbauer matrix polynomials and second order Matrix Differential Equations. Div. Math. 12(2), 101-115 (2004)

29. Sayyed, K.A., Metwally, M.S., Batahan, R.S.: On generalized Hermite matrix polynomials. Electron. J. Linear Algebra 10, 272-279 (2003)

30. Shehata, A.: A new extension of Gegenbauer matrix polynomials and their properties. Bull. Inter. Math. Virtual Inst. 2, 29-42 (2012)

31. Shrestha, N.B.: Polynomial associated with Legendre polynomials. Nepali Math. Sci. Rep. 2(1), 1-7 (1977)

32. Sinha, S.K.: On a polynomial associated with Gegenbauer polynomial. Proc. Nat. Acad. Sci. India Sect. A 54, 439-455 (1989)

33. Srivastava, H.M., Brenner, J.L.: Bounds for Jacobi and related polynomials derivable by matrix methods. J. Approx. Theory 12, 372-377 (1974)

34. Srivastava, H.M., Manocha, H.L.: A Treatise on Generating Functions. Halsted Press, Wiley, New York (1984)

Publisher's Note Springer Nature remains neutral with regard to jurisdictional claims in published maps and institutional affiliations. 